\title{
XX.
}

(Aus der medicinischen Klinik des Herrn Prof. Erb in Heidelberg.)

\section{Beitrag zur Aetiologie, Symptomatologie und Therapie der Tabes dorsalis.}

Von

\author{
Dr. J. Hoffmann, \\ Assistenzarzt.
}

\begin{abstract}
$\mathrm{K}_{\mathrm{a}}$ aum eine andere Krankheit ist in den letzten Jahren so reichlich bearbeitet worden, als die Tabes dorsalis, sowohl in ätiologischer, symptomatologischer wie auch pathologisch-anatomischer Beziehung. Die Therapie kam dabei, wie bei so manchen anderen cbronischen Krankheiten, meist stiefmütterlich weg, weil man nicht viel Gutes davon zu sagen weiss. Die folgenden Fälle enthalten manches Neue und Interessante, was ich der Veröffentlichung für werth halte.

Was die Aetiologie betrifft, so haben uns Fournier und Erb auf statistischer Basis die unumstössliche, ron mancher Seite immer noch angefochtene Thatsache kennen gelehrt, dass die Syphilis „eine der wichtigsten, weun nicht die wichtigste Bedingung für das Entstehen der Tabes ist". Neuere Statistiken beweisen die Richtigkeit dieses Satzes auf's Neue und werden manchen Gegner der Syphilisätiologie der Tabes langsam bekehren. Dass es neben der Syphilis noch einige andere Momente giebt, denen die Tabes folgen kann, haben dieselben Autoren, die die Syphilis als das vorwiegendste ätiologische Moment der Krankheit hinstellten, stets anerkannt. Unter diesen Ursachen spielt auch das Trauma eine gewisse Rolle. $O b$ und in wie weit in dem nachfolgenden Falle der Entstehungsmodus als Trauma betrachtet werden kann, dies will ich erst erörtern, nachdem ich die Krankengeschichte vorausgesehickt habe.
\end{abstract}




\section{Fall r.}

Erkältungen, schwere Arbeit. Directer Anschluss der Krankheit an gleichartige Frschütterungen (6000-10,000 täglich) des Körpers im Sommer 1883. Vom Winter 1883-1884 ab Gürtelgefühl. Ianoinirende Schmerzen, Schwäche in den Beinen, Augenmuskellähmung, gastrischë Krisen. Frontalschmerz, Ohrensausen, Schwerhörigkeit. - Myosis, refiectorische Pupillenstarre, Schwerhörigkeit mit Hyperaesthesie der N. acustioi gegen den galvanischen Strom, Salivation, gastrisohe Krisen, Enterorrhoen, Fehlen aller Zähne des Oberkiefers, Abfallen der Zehennägel, Ataxie, verlangsamte Schmerz- und Temperaturleitung der Beine, Cystitis, Urinträufeln.

Gustav Reichensberger, 47 Jahre alter, verheiratheter Fabrikarbejter von Wieblingen, rec. 21. August 1886, entlassen 10. December 1886.

Keine Nervenkrankheiten in der Familie; keine syphilitische Infection; in zwei Ehen, in dener or 11 Kinder zeugte, kein Abort. Sexuelle Excesse werden in Abrede gestellt; auch war er selbst nie "nervös".

1871 litt er 4 Tage lang an dysenterischen Erscheinungen, 1883 an Erysipel der linken Hand.

Er arbeitete schwer in einem heissen Raum mit sehr viel Zugluft nnd zog sich dabei öfter leichte Erkältungen zu. Patient selbst führt sein Leiden auf härfige und heftige Erschütterungen des Bauches und des ganzen Körpers zurück, die er bei seiner Arbeit täglich auszuhalten hatte. Das genauere Examon über diese Erschütterungen ergiebt Folgendes: Patient beschäftigte sich mit der Herstellung (dem Schlagen) von Nägoln aus mehrere Millimeter dicken Blechplatten. Ungefähr $1 / 4$ Jahr vor seiner Erkrankung wurde eine neue Art des Blechschneidens eingeführt, die darin bestand, dass die Blechplatten durch einen niederfallenden keilförmigen Hammer auf einer ambosartigen Unterlage durchgeschlagen wurden. Damit die Platte nicht auswich, stemmte sich der Kranke mit aller Rraft seines Körpers mit dem Unterleib gegen dieselbe und hatte so die dureh den Fall hervorgerafene Erschütterung derselben auszuhalten. Anfangs arbeitete or im dritten Stock, dessen Fussboden durch den gewaltigen Hammersehlag, "den man im ganzen Dorfe hörte“, mit in Schwankungen gerieth; später wurde die Werkstatt zu ebener Erde verlegt. Der Shok der Blechplatte sei so gewaltig gewesen, dass die anderen Arbeiter ihn nicht ablösen konnten, weil sie die Kraft zum Widerstand gegen die Platte nicht hatten. Er schnitt täglich 40 Blechplatten in 150-250 Stücke, was, jeden Schlag einfach gerechnet, pro Tag 6000 bi is 10,000 Erschütterungen des Körpers beträgt, ganz abgesehen von den Sohwankungen des Bodens.

Ein Vierteljahr, nachdem er sich in dieser Weise beschäftigt hatte, begann im Winter 1883/84 die jetzige Krankheit, deren Hauptsymptome bis jetzt luru folgende waren und sind: Gürtelgefühl um das Mesogastrium, 
lancinirende Schmerzen in deu Beinen mit unwillkürlichen Zuckun$g \theta n, S c h w a ̈ c b \theta$ und Unsicherheit der Beine, welche letztere im Dunleln sich steigert; $A$ bnahme der Schweissproduction, die er ron Kindheit an recht reichlich batte; A usfallen der Zähne, auch solcher, die ganz gesund waren. Seit den sechziger Jahren, wo er die beiden Schnoidezähno durch einen Fall verloren batte, waren keine mehr ausgegangen. Er konnte sie nun selbst mit den Fingern herausziehen, ohne dass es ihn schmerzte. April 1884 Ptosis des linken oberen Augenlides mit Deviation des linken Bulbus nach aussen. Von Januar 1886 ab Unfähigkeit zu gehen, Incontinentia urina $\theta$, hartnäckige Verstopfung, alle 3-4 Wochen sich wiederholende Gastralgien mit grünlichem reichlichem Erbrechen, was 3-4 Tage dauert. Hypălgesie der Beine. Drückender, fast continuirlicher Frontalschmerz. Seit ungefähr 2 Jahren Sausen, Brausen und Pfeifen in beiden Ohren mit Sohwerhörigkeit.

- Therapie symptomatisch.

\section{Status praesens.}

Blasses, mageres Individuum von kräftigem Skeletbau.

Die inneren Organe normal.

Myosis mitreflectorischer Pupillenstarre; Paresis levator. palpebr. super. oc. sin. mit Lähmung des M. rectus intern. oc. ejusdem; Augenhintergrund normal. Fehlen aller Zäbne des Oberkiefers mit Atrophie des Alveolarfortsatzes. Zunge feucht, normal in jhren Bewegungen. Hörfähigkeit beidersoits stark herabgesetzt; Geruch und Geschmack gut.

Fehlen der Sebnenreflexe der oberen Extremitäten bei gut erhaltener grober motorischer Kraft, intacter Sensibilität, Coordination und bei gutem Muskelsinn.

Die unteren Extremitäten sind sehr mager, aber kräftig. Starke Ataxie und beträchtliche Störung des MuskeIsinns.

Von der 5. Rippe abwärts Tastempfindung bis zu don Fusssoblen herab stark herabgesetzt; Hypalgesie mit bis zu 2 Secunden verlangsamter Schmerzleitung. Temperatursinn von den Knien abwärts schlecht, fast erloschen und dabei dje Leitung der Empfindung verlang. samt. An der Fusssohle wird warm und kalt ziemlich sicher unterschieden.

Patellar- und Achillessebnenreflex versehwunden.

Plantarreflex schwach; Cremaster- und Bauchreflex fehlen.

Cystitis, Urinträufeln; Blase leer.

Nägel der 1., 2. und 3. Zehe des linken Fusses verkrüppelt, im Abstossen begriffen.

Von den während des Hierseins des Kranken notirten anamnestischen Angaben und beobachteten Symptomen seien noch folgende erwähnt: P assagere 1-2 tägige Pulsbeschleunigung auf $100-108$, während für gewöhnlich nur 76-84 zu zählen waren. Mehrtägige, alle paar Wochen 
wiederbolende Diarrhoen mit Tenesmus. Beim Lachen und Sprechen entsteht regelmässig dăs Gürtelgefühl und schwindet wieder mit dem Aufhören zu reden. Spannen in der Stirn und in der rechten Wange. Des Nachts bat or Schwierigkeit, die Kiøfer auseinander au bringen, wenn or Wasser trinken will.

27. October. Klagt über Speichelfluss; er bringt den Mund nie rein von "dem zähen Speichel"; lancinirende Schmerzen etc.

Sehr starke Hyperästbesie beider Acustici gegen den galva. nischen Strom mit paradoxer Reaction.

30. August 1887. Um Ostern, Pfingsten und vor 14 Tagen gastrische Krisen von 4tägiger Dauer. Abnahme des Gedächtnissos „durch die heftigen Schmerzen".

Es bedarf wohl keiner weiteren Begründung, dass es sich hier um einen Fall von Tabes dorsalis handelt. Ohne die gewöhnlichen Symptome zu recapituliren, will ich nur aufmerksam machen anf das Nebeneinandervorkommen einer Reihe seltener Erscheinungen wie: das Ausfallender Zähne, das Abgestossenwerden der Zehennägel, die verminderte Schweissproduction, die Salivation, die gastrischen Krisen, die Enterorrhoen, die herabgesetzte Hörfähigkeit mit beträchtlicher Hyperästhesje beider Nerv. acustici gegen den galvanischen Strom und endlich die selten beobachtete Verlangsamung der Temperaturleitung.

Interessanter erscheint mir die Aetiologie. Die Krankheit entwickelte sich im Anschluss und wobl in Folge von sehr zahlreichen, täglich bis in die Tausende zählenden gleichartigen Erschütterungen des Körpers, besonders des Abdomens. Mit einem gewöhnlichen Trauma lässt sich dieser Vorgang nicht leicht auf gleiche Stufe stellen. Er ähnelt vielmehr der Art und Weise, auf die Westphal bei Meerschweinchen Epilepsie erzeugte, dem Goltz'schen Klopfversuch, sowie der Entstehung des als Railway spine bezeichneten Leidens und erinnert andererseits an die Versuche von Mendel*) und Fürstner*), die bekanntlich Hunde auf einer Drebscheibe mit dem Kopf nach der Peripherie befestigten und die Drehscheibe in rotirende Bewegung setzten. Mendel liess die Hunde täglich 4-5 Minuten lang drehen mit einer Geschwindigkeit von

* Mendel, Ueber paralytischen Blödsinn bei Hunden. - Ref. im Neurol. Centralbl. 1884, S. 227.

**) Fürstner, Ueber experimentelle Untersuchangen im Bereich des Centraluervensystems; Vortrag gehalten in der 59. Versammlung deutscher Naturforscher und Aerzte in Berlin. 
100-110 Umdrehungen in der Minute. Er setzte dieses Verfahren so lange fort, bis die Thiere Verlust des Muskelgefühls in den unteren Extremitäten zeigten, was meist $z$ wischen dem 10.-12. Tage der Fall war. Dann überliess er die Hunde bei guter Ernährung ihrem Schicksal und sah sie in einen Zustand gerathen, den er mit der Paralyse der Irren beim Menschen auf eine Stufe stellt. Die active Hyperämie des Gehirnes hält er für wesentliche Bedingung zur Entstehung der Krankheit. Fürstner hat bei derselben Lagerung des Thieres auf der Drehscheibe nur $1-2$ Ninuten am Tage die Drehungen in möglichst geringer Intensität vorgenommen, öfter auch 60-80 Drehungen in der Minute gemacht. Thiere, die $3 / 4$ und $5 / 4$ Jahre so gedreht worden waren, zeigten doppelseitige primäre Degeneration der Seitenstränge und eines bestimmten Abschnittes der Hinterstränge, Veränderungen der Nn. optic.*) und ähnliche Veränderungen im Gehirn, wie sie Mendel gefunden hatte.

Unser Kranker merkte die ersten Erscheinungen seiner Krankheit, nachdem er unter sonst gleich gebliebenen Verhältnissen 1/4 Jahr lang täglich mehrere Tausende gleichartige Erschütterungen ausgehalten hatte. Ich begnüge mich damit, diesen Fall als einen Parallelfall za den experimentellen Resultaten der genannten Autoren hinzustellen, olne mich auf hypothetische Erörterungen einzulassen.

\section{Fall II.}

1881 Syphilis; sonst keine ätiologischen Momente. Frühjahr 1885 Urinbesohwerden, von Eerbst 1885 ab Obstipation, gastrische Krisen, Müdigkeit der Beine. - Hypalgesie der Füsse und Unterschenkel, Fehlen der Patellar- und Achillessehnenreflexe; Urinträufeln, Cystitis. Gastrische Krisen von ausserordentlich langer Dauer, Hyperacidität des Magensaftes mit Hypersecretion, Singultus, Irregularität der Herzaction und der Athmung, epileptisohe Anfälle. Träge Reaotion der erweiterten Pupillen, lancinirende Schmerzen.

Christian Zimmer, lediger, 27 Jahre alter Kaufmann von Guntersblum, befand sich rom 7 . September 1886 ab ein volles halbes Jahr auf der medicinischen Abtheilung in Behandlung.

Aus einer gesunden Familie stammend, machte er nur die Kinderkrankheiten und einmal Erysipel durch; wegen „allgemeiner Körperschwäche" wurde or militärfrei. Traumata, Erkältungen, grössere Strapatzen hat er nicht überstanden.

*) Fürstner, Ueber Veränderungen an den Nn. optic., Vortrag gehalten in der XII. Wanderversammlung der südwestdeutsohen Neurologen und Irrenärzte in Strassburg. 1887. 
1881 syphilitischo Infection (harter Schanker, Hautsyphilid, Angina specifica); Jodkali, Pillen.

Seit $1 \frac{1}{2}-1 \frac{3}{4}$ Jahren träge Urinentleerung bei Urindrang, Nachts öfter Enuresis; damit gleichzeitig leichte Trübung des Urins.

Von Herbst 1885 ab Obstipation und AnfäIle von Erbrechen. Die Brechanfälle kehrten bis vor $1 \frac{1}{2}$ Monaten alle $3-4$ Wochen ohne bekannie Ursache wieder; sie danerten meist von Morgens 10 Uhr bis Nachmittags 4 Uhr. Das Erbrochene schmeckte intensiv sauer, bestand nur aus hellem Schleim und gallig gefärbter, etwas zäher Flüssigkeit; ni $\theta$ wurde:l Speisen miterbrochen. Durștgefühl während der Anfälle sehr gesteigert; grosse Uebelkeit, Magendruck ohne besonderen Schmerz, Abgeschlagenheit, kalte Füsse. Seit 6 .Wochen tägliches Eintreten dieser Anfälle von Morgens früh oder schon Nachts bis gegen Mittag, 'dann konnte er wieder essen.

Mit den täglichen Magenkrisen fühlto or starke Müdigkeit in den Beinen und fast immer kalte Füsse.

Andere auf eine spinale oder cerebrale Erkrankung bindeutende Symptome fehlen bis jetzt vollständig.

Am 24. Juli 1886 wurde er nach Liebenzell geschickt wegen seines "Magen- und Blasenleidens". Er bekam da eine Salzsäuremixtur verordnet, bekam aber schon nach dem 2. Esslöffel voll solches Brennen und Drücken in der Magengegend mit Brechneigung, dass or sich entschieden weigerte, die Arznei weiter zu nehmen.

\section{Status praesens.}

Grosser, schlanker, etwas anämischer Mensch, mit schwach entwickelter Muskulator and geringem Fetipolster.

Die psychischen Functionen normal.

Die $\mathrm{P}$ apillen ziemiich weit, rund, beide gleich weit, reagiren prompt auf Licht und Accommodation. Weder an den Augenmuskeln, noch sonst eine Störung der Motililät oder des Gefühls des Gesichts.

Die oberen Extremitäten verhalten sich normal. Die Sehnenreflexe sind lebhaft; links ist der Tricepsreflex otwas schwächer als rechts.

Die $\mathrm{B} \theta \mathrm{in}$ e sind frei von vasomotorischen und trophischen Störungen; die Füsse otwas sohweissig, nicht kalt.

Tast- und Temperatursinn gut. Die Schmerzempfindang ist am Fuss und Unterschenkel beiderseits etwas a $\mathrm{g}$ estumpft; keine evidente Verlangsamung der Schmerzleitung. Plantar-, Cremaster- und Abdominalreflex normal; weder Ataxie, noch Muskelsinnstörung, die mechanisehe Muskelerregbarkeit ist normal; ebenso die grobe motorische Kraft. Die Sehnenreflexe $\mathrm{f} \theta \mathrm{hle} \mathrm{n}$.

Die inneren Organe normal; nur eine Cystitis besteht.

8. September. Erbrechen einer reichlichen Menge fast farbloser, leicht sohleimiger, speisefreier Flüssigkeit von stark saurem Geruch und ebensolcher 
Reaction. Intensive Salzsäure-Reaction. Das Erbrechen dauert von 6 Uhr Morgens bis gegen Mitlag. Am Nachroittag Wohlbefinden.

Temperatur and Puls normal.

9. Seplember. Wohlbefinden. Träge Urinentleerung and häufig Urinträufeln.

10. September. Seit heute Nacht 2 Uhr Erbrechen; das erst Mittags 2 Uhi sistirt. Uebelkeit, Brennen in der Magengegend, gesteigertes Durstgefühl. Das Erbrochene stark HCl-haltig.

13. September. Zur selbigen Zeit wie seither täglich gastrische Krisen. Die einzelnen Brechacte mit Entleerung des Magens liegen meist 3 bis 4 Stunden auseinander. Magendruck; $A$ bgeschlagenheit; grosse Uebollueit, Durst.

Vom 14.-22. September (excl, 21. September) tägliches Frbrechen in gesehilderter Weise and unter denselben Symptomen. Das Erbrochene ent. hält von der Abends genommenen Nahrung (Floisch, wenig Brod und Wein), nie Reste, sieht häufig leicht gallig gefärbt aus.

Die Probe auf $\mathrm{HCl}$ gelingt stets, nicht die auf Milch- und Buttersäure.

\begin{tabular}{|c|c|c|c|}
\hline Datum. & $\begin{array}{c}\text { Erbrochenes. } \\
\text { Cbotm. }\end{array}$ & $\begin{array}{c}\mathrm{HCl} \text { - Gehalt. } \\
\text { pCt. }\end{array}$ & Pulsfrequenz. \\
\hline $\begin{array}{c}\text { September } \\
14 . \\
15 . \\
16 . \\
17 \\
18 . \\
19 . \\
20 .\end{array}$ & $\begin{array}{l}850 \\
750 \\
600 \\
1600 \\
750 \\
750 \\
600 \text { und } \\
900 \\
=1500\end{array}$ & $\begin{array}{c}-\overline{0} 19 \\
0,419 \\
0,291 \\
0,279 \\
0,200 \\
0,240 \\
\overline{0,2125}\end{array}$ & $\begin{array}{c}80-84 \\
80 \\
80 \\
84 \\
80 \\
84 \\
\\
84\end{array}$ \\
\hline $\begin{array}{l}21 . \\
22 . \\
23 . \\
24\end{array}$ & $\begin{array}{l}\text { Nichts } \\
1350 \\
\text { Nichts } \\
750\end{array}$ & $\begin{array}{c}\overline{0,109} \\
-\end{array}$ & $\begin{array}{l}84 \\
84 \\
80 \\
80\end{array}$ \\
\hline
\end{tabular}

Das zuletzt Erbrochene enthält nur Fetttröpfchen; keine Speisereste anderer Art; keine Sarcine.

Vom 25. September bis 20. November keine einzige gastrisohe Krise. Der Kranko sieht viel besser aus, als beim Eintritt in's Krankenhaus und klagt über folgende Beschwerden:

Urinträufeln und häufige Urinentlecrung in der Nacht; Obstipation; selten laneinirende Schmerzen im linken Bein und im linken Vorderarm, Brennen in den Fussoblen. Am 19. October häufiger Stuhldrang und Reissen durch den Damm nach der Uretbra. 
28. October. Die Pupillen sind ziemlich weit, reagiren aber auf einfallendes Licht etwas träge. Leichtes Schwanken beim Stehen mit geschlossenen Augen.

Eine zwei Tage nach der Aufnahme des Kranken oingeleitete Inunctionscur mit Ung. hydrarg. cin. wird am 11. Norember ausgesetzt, nachdem er 240,0 Unguent. cin. eingerieben. Er befand sich dabei subjectiv ganz wohl, hatte keinerlei mercurielle Erscheinungen. Zunahme des Körpergewiohts vom 30. September bis 10 . November um 2,5 Kilo.

14. November. Pulsbeschleunigung auf 104 Schläge in der Minute; dabei fleckige Röthung der Haut und leichte Schweissbildung.

17. November. Klinische Vorstellung. Diagnose: Tabes dorsalis mit gastrischen Krisen.

20. November. Neunmalige Urinentleerung in der Nacht. Er klagt hente seit Sistirung des Erbrechens zum ersten Male wieder über Uebelkeit und Unbehagen.

Temperatur 36,7; Pulsfrequenz 84. Um 10 $\frac{1}{2}$ Uhr beginnt Erbrechen unter Druckgefühl im Hypogastrium. Das zuerst Erbrochene entliielt noch etwas Kaffee beigemischt; das spätere war eine fast farblose, leicht schleimige, intensiv sauer reagirende Flüssigkeit von „sehr saurem Geschmack “. Der Puls nur während des Brechactes an Frequenz wechselnd. Der Urin ist dabei intensiv sauer.

21. November. Das Erbrechen sistirte von Nachts 12 Uhr bis heute Morgen $8 \mathrm{Uhr}$. Reichliches Erbrecben, trotzdem der Kranke seit 24 Stunden keinen Tropfen Flüssigleit zu sich genommen, noch feste Nabrung. Klage über Druck im Unterleib, Abgeschlagenheit.

Charakteristische Jactation; häufiges Gähnen, Salivation. Gesichtszüge verfallen.

Puls stark gespannt bei weiler, voller Radialarterie, unregelmäs sig, nicht aussetzend. Unregelmässige Respiration, so dass auf 10 Secunden bald nur eine, bald $4-5$ Athmungen kommen.

Pulsfrequenz 100 , Temperatur $37,1^{\circ} \mathrm{C}$.

Das Erbrochene leicht gallig gefärbt, intensiv sauer. Der Urin sauer, 600 Cbetm. in 24 Stunden.

Um 2 Uhr hört das Erbrechen auf, der Kranke geniesst etwas Milch mit Selterswasser.

Bei der Abendvisite $\mathrm{Puls}$ beschleunigt, 120 in der Minute, regelmässig. Eingenommenheit des Kopfes, Mattigkeit. Salivation, seitdem er erbricht. Die genossene Mileh kommt geronnen zurück.

22. November. Schlaf schlecht. Zeitweise "klemmender" Schmerz im Hypogastrium, der nach der Magengrube heraufzieht und mit Erbrochen endet. Stuhl spärlich.

Gesicht verfallen, Aufstossen, Gähnen. Erbrechen von 12-1 Uhr Nachts und von 6 Ohr ab heute frïh.

Puls gespannt, 108, otwas unregelnässig, nicht aussetzend. Respi- 
ration regelmässig. Rascher Wechsel der Gesichtsfarbe und fleckige Röthung des Rumpfes.

Urinmenge 500 Cbotm., specifisch. Gewicht 1023.

Ord.: Potio Riveri. - Eismilch and Eispillen.

11 Uhr Morgens: Der Kranke nimmt bei vollem Bewusstsein die Arznei, wird kaum 5 Minuten später plötzlich ganz blass and athmet kaum sichtbar.

Gesicht blass, ungemein verfallen; Lippen und Hände bla u, letztere trotzdem warm. Puls an Frequenz rasch wechselnd meist gegen 120, selten sinkend bis 108; nur einige Male kommt auf $4--5$ Secunden je nur 1 Pulsschlag und sogar weniger; dann 50-60 Sohläge in der Minute. Respirationsfrequenz 6-30 in der Minute; keine Regelmässigkeit in der Zuund Abrahme der Puls- und Respirationsfrequenz. Der Puls ist qualitativ ziemlich normal. - Temperatur leicht erhöht, $37,7^{\circ} \mathrm{C}$.

Der Kranke ist bew usstlos, die Pupillen sind verengert, reactionslos. In diesem Zustande befindet sich der Kranke ungefähr 5 Minuten. Unter starker Röthung des Gesichts lrommt er dann zu sich und weiss von dem Vorgefallenen nichts.

12 Uhr: Erbrechen; von 1-2 Uhr Singultus und rascher Wechsel der Gesichtsfarbe.

3 Uhr: Erneuter Anfall von Bew usstlosigkeit mit verengerten, reactionslosen Pupillen, Unregelmässigkeit der Atbmung und der Herzaction. Der Puls erreicht zeitweise eine Frequenz von 140. Cyanose der Hände and der Lippen. Einige Secunden lang olonische, schwache Zuckungen der kleinen Nackeńmuskeln; dieselben wiederholen sich in diesen Muskeln 2-3 Mal, dann treten clonische, etwas stärkere Zuckungen in den M. pectoralis dext. et sin. und auch der SchuIterheber hinzu. Auch dieser Anfall verschwindet unter Röthung des Gesichts nach 5 Minuten.

4 Uhr: Singultus; Gähnen. A. radial. sehr weit; Puls stark gespannt.

Urin intensir sauer, cystitisch.

$6 \frac{1}{2}$ Uhr: Singultus. Pulsfrequenz 96.

23. November. Schlaf unruhig. Uebelkeit, Brechreiz. Gesicht geröthet; Puls wochselnd, 96. Pupillen weit, reagiren auf Licht.

Vom 23.-28. November kein Erbrechen. Die Klagen des Kranken sind: Brennen im Magen, Durst, Klemmen" im Unterleib. Speichelfluss, der besonders in der Nacht sehr störend ist. Urinträufeln, sehr träge und erschwerte Urinentleerung.

Eingenommenheit des Kopfes; Abgesehlagenheit, "elendes" Gefühl. - Vom 25. November ab hört der Wechsel in der Pulsfrequenz auf, auch die Gesichtsfarbe wechselt nicht mehr wie vorher. Urins.

Urinmenge $750-1250$ Cbetm,; Cystitis bei saurer Reaction des

Vom 28. November 1886 bis 24. Januar 1887 täglicbes Erbrechen, ausgenommen wenige Tage, die zeitlich auseinander liegen und an 
denen unabhängig von gegebenen Medicamenten das Erbrechen wegblieb bei Fortdauer des subjectiven "Elendsgefühls".

Die Erscheinungen waren stets die geschilderten, Anfälle von Bewasstlosigkeit und clonischen Zuckungen kehrten nicht wieder.

Das Erbrochene war häufig gefärbt, reagirte intensiv sauer, gab mit Methylviolett, Tropaeolin und Carbolsäure - Liqu. ferr. sesquichloridlösung stets die $\mathrm{HCl}-\mathrm{Re}$ action. Mittelst des letzten Reagens fiel die Untersuchung auf Milch- und Buttersäure, so oft dieselbe rorgenommen wurde, negativ aus. Herr Apotheker Brunner hatte die Freundlichkeit, die Titrirung des Erbrochenen mit Normalkalilauge auszuführen und nach dem Cahn-v. Meringschen Verfahren die Säure zu bestimmen. Er vermisste auch mit dieser Probe Milch- und Buttersäure bei mehreren darauf gerichteten Untersuchangen. Herrn Brunner spreche ich auch an dieser Stełle meinen verbindlichsten Dank aus.

Das am 20. November znerst Erbrochene, 400 Cbotm, enthielt 0,146 pCt. HCl; das Erbrochene rom 20. November Nachmittags enthielt keine Beimischung von Kaffee mehr; am 21. November Morgens erbrach Patient zum letzten Mal, 22 Stunden, nachdem or Kaffeo getrunken batte, während welcher Zeit er auch keinen Tropfen Flüssigkeit zu sich genommen hatte. Die Nenge dieses Erbrochenen betrug $1000 \mathrm{Cbctm}$, und enthielt 0,1 pCt. HCl.

\begin{tabular}{|c|c|c|}
\hline Datum. & $\begin{array}{c}\text { Erbrochenes } \\
\text { in Cbctm. } \\
24 \text { stündige } \\
\text { Menge. }\end{array}$ & pCt. $\mathrm{HCl}$. \\
\hline $\begin{array}{c}\text { November } \\
29 . \\
30 . \\
\text { December } \\
2 . \\
3 . \\
4 . \\
5 . \\
6 . \\
7 . \\
8 . \\
9 . \\
10 . \\
11 . \\
12 . \\
13 . \\
14 . \\
15 . \\
16 . \\
17 . \\
18 . \\
19 . \\
20 . \\
21 .\end{array}$ & $\begin{array}{c}900 \\
750 \\
\\
250 \\
200 \\
750 \\
300 \\
500 \\
\text { Nichts } \\
250 \\
300 \\
300 \\
750 \\
750 \\
1000 \\
300 \\
? \\
? \\
1000 \\
250 \\
1000 \\
? \\
1000\end{array}$ & $\begin{array}{c}0,24 \\
0,32 \\
\\
0,31 \\
0,18 \\
0,21 \\
0,33 \\
0,21 \\
-\overline{7} \\
0,249 \\
0,255 \\
0,351 \\
0,25 \\
0,233 \\
0,24 \\
0,277 \\
0,219 \\
? \\
0,219 \\
0,21 \\
0,31 \\
? \\
0,17\end{array}$ \\
\hline
\end{tabular}




\begin{tabular}{|c|c|c|}
\hline Datum. & $\begin{array}{l}\text { Erbrochenes } \\
\text { in Cbctm. } \\
24 \text { stündige } \\
\text { Menge. }\end{array}$ & pCt. HCl. \\
\hline $\begin{array}{c}\text { December } \\
23 . \\
25 . \\
27 . \\
28 . \\
30 . \\
31 . \\
\text { Januar } 1887 \\
2 . \\
3 . \\
4 . \\
5 . \\
6 . \\
7 . \\
8 . \\
9 . \\
10 . \\
11 . \\
12 . \\
19 . \\
24 .\end{array}$ & $\begin{array}{c}500 \\
750 \\
300 \\
500 \\
500 \\
300 \\
\\
300 \\
250 \\
300 \\
300 \\
? \\
200 \\
300 \\
\text { Nichts } \\
800 \\
? \\
250 \\
? \\
1000\end{array}$ & $\begin{array}{c}0,314 \\
0,219 \\
0,219 \\
0,277 \\
0,197 \\
0,248 \\
\\
0,255 \\
0,102 \\
? \\
? \\
? \\
0,219 \\
0,146 \\
-\overline{182} \\
0,292 \\
0,182 \\
0,219 \\
0,146\end{array}$ \\
\hline
\end{tabular}

Während der ganzen Dauer dieser mehrwöchentlichen gastrischen Krise litt der Kranke an den erwähnten Urinbeschwerden, an Brennen und Druck im Unterleib, Magendruck, Uebelieit; Obstipation. Abgeschlagenheit, Eingenommenheit des Kopfes, Somnolenz mit Jactation. Der Leib war fast immer tief eingesunken und nicht druckempfindlich. "Mehlspeisen liegen schwer im Magen", während Feisch gut verdaut wird. Lancinirende Schmerzen hatte er während dieser Zeit nur zwei Mal im Schienbein.

Sehr häufig orbrach er noch des Morgens, nachdem er um 7 Uhr Abends die letzte Nahrung und die letzte Flüssigkeit zu sich genommen hat, wovon das Erbrochene nichts enthielt.

Vom 5. December ab machte der Kranke nochmals eine Schmiercur von 30 Einreibungen à 3,0 Ung. cin. durch. Morphium brachte nur vorübergehend Linderung. Vom 3.-13. Januar $1887 \mathrm{kam}$ Antifebrin in steigender Dosis in Anwendung; er nahm $3 \mathrm{Mal}$ täglich 0,25 bis $3 \mathrm{Mal}$ täglich 1,0 Antifebrin. Er fühlte sich dabei vorübergehend besser als vorher; das Erbreehen hörte nicht auf und der $\mathrm{HCl}$ Gehalt des Erbrochenen war auch nicht nennenswerth verändert. - Am raschesten half ihm bei dem starken Magendruck und Magenbrennen ein kalter Priessnitz'scher Umschlag. Vom 22. Januar ab galvanische Bebandlung und Karlsbader Salz. Von diesem Tage ab wurde der Urin klarer bei dem Fortbestehen der Urinbe- 
schwerden; er erbrach nur noch am 24. Januar. Am 27. Januar war der bis dahin oystitische Urin ganz klar geworden.

Von der Zeit ab, wo das Erbrechen wegblieb, schienen an dessen Stelle lancinirende Schmerzen von grösserer Heftigkeit getreten zu sein. Sie waren localisirt in den Unterschenkel, den linken Arm und in den Damm; die letzteren waren besonders unangenehm. Der Urin blieb klar.

13. Februar. Ord,: Kal, jod. 5,0:150,0 Aqu. 3 Mal täglich 1 Ess löffel zu nehmen.

22. Febraar. Der Kranke sollte heute entlassen worden, da stellte sich in Folge der psychischen Erregung (?) das Erbrechen wieder ein mit all den fräheren Begleitorscheinungen; a uch die Cystitis kebrte wieder.

7. März. Der Kranke wird heut auf Wunsch entlassen. Er erbrach seither fast täglich, das Erbrochene wurde auch jetzt wieder regelmässig untersucht und enthielt $0,058-0,35 \mathrm{pCt}$. HCl. Linderung spürte der Kranke auf die Application des Priessnitz'schen Umschlages, während er die Eisblaso nicht vertrug, auf Karlsbader Salz und aufCocain 0,05 zwei Mal täglich innerlich, in welcher Verabreichung es besser wirkte, als wenn es injicirt wurdo.

$$
\text { Status praesens. }
$$

Pupillen weiter als normal, träger auf Licht reagirend als in der Norm. Fehlen der Sehnenreflexe der unteren Extremitäten. Hyperästhesie gegen Kälte von der VIII.-XII. Rippe, gürtelförm $\gamma$ und an der Innenflächeder Oberschenkel, geringe Herabsetzu. g der Sehmerzempfindung an den Unterschenkeln. Cystitis.

Keine Ataxje, keine Muskelsinnstörung.

Mitte Juni. Nach einer Mittheilung des behandelnden Arztes dauert das Erbrechen bis jetzt fort, setzt meist jeden 2. oder 3. Tag aus.

\section{Fall IXI.}

Sohwere Arbeit, Frkältungen, zweiter Mann syphilitisch. 1881 lanoinirende Schmerzen, Paraesthesien, 1883 unsicherer Gang. Sommer 1885 gastrische Krisen, Gürtelgefühl, Analgesie und Sohwäche der Beine. Reflectorische Pupillenstarre, Hypalgesie der Arme und des Rumpfes, Analgesie der Beine mit verlangamater Schmerzleitung, Ataxie der Beine und des reohten Armes, lancinirende Schmerzen, Fehlen der Sehnenreflexe, Cystitis. Intercurrente, fieberhafte \#rkrankung. Gastrische Krisen. epileptische Anfälle.

Katharina We is s kapp, 35 Jahre, verheirathete Maurersfrau von Solilierbach, rec. 16. Juni 1887, entlassen 10. August 1887.

Weder neuropathische, noch überhaupt herediäre Belastung. Menses unregelmässig. Aus erster Ehe sind zwei gesunde. Kinder da; in der zweiten gebar sie fünf gesunde Kinder, von denen das jüngste, $2 \frac{1}{2}$ Monate alte während des Spitalaufenthalts der Mutter starb; kein Abort. 
Als ursächliche Momente der jetzigen Krankheit werden angegeben: schwere Arbeit (als Dienstmagd) und Erkältungen bei der Beschäftigung in der Cigarrenfabrik. Nach dreijähriger Ehe mit dem zweiten Manne, der zugestebt in seinem 17. Lebensjahre "wegen Tripper eine Schmierou $\mathbf{r}^{\text {", und }}$ zwar in der Heidelberger Klinik durchgemacht zu haben, zeigten sich im Jahro 1881 lancinironde Sohmerzen und Parästhesien in den Beinen, zwei Jahre später Abgeschlagenheit, atactischer Gang, Unsicherheit im Dunkeln. Seit einem Jahre geht sio am Stock; seit vorigem Herbst leidet sie an Parästhesien im Ulnarisgobiet.

Im Sommer $1885 \mathrm{lag}$ sie 4 Wochen lang zu Bett wegen gastrischer Krisen, die während dieses Zeitraums alle $3-4$ Tage kamen und dann ebenso lange wegblieben. Im Juli 1886 dauerten sie bereits 16 Wochen mit mit grösserer Heftigkeit. Am 10. Mai dieses Jahres erneute Attaque mit täglichem Erbrechen. Dieselben verlaufen unter Brennen im Magen mit heftigem wühlendem, krampfartigem Schmerz in der epi-und hypogastrischen Gegend und unter unstillbarem Durst. Stuhl während der gastrischen Krisen angehalten.

In letzter Zoit Gürtelgefühl, Analgesie der Boine; seit Jahren Urindrang und passagere Uninretention, ferner Schwäche des Sphincter ani, abwechseInd mit hartnäckiger Obstipation. - Schwierigkeit, beim Nähen die Nadel richtig zu führen.

Cerebrale, bulbäre Symptome, Augenmuskellähmungen, Polyurie etc. fehlter stets.

\section{Status praosens.}

Grosse, schlanke, magere Frau mit schmutzig blassgelber Gesichts- and Hautfarbe. Augen eingesunken; verfallene Gesichtszüge. Sïe antwortet träge und mit matter Stimme.

Pupillen unregelmässig geformt, weit, reflectorisch starr. Gehirn und Gehirnnerven verhalten sich sonst normal. Hypalgesie der oboren Extremitäten und Fehlen der Sehnenreflexe bei guter Tast- und Temperaturempfindung. Leichte Ataxie und obensolche Störung des stereognostischen Sinns des rechten Arms resp. der rechten Hand.

Hypalgesie am Rumpf.

Fast complete Analgesie der Beine bei im Ganzen gut erhaltener Temperaturempfindung und geringer Herabsetzung der Tastempindung des recbten Fusses und Unterschenkels. Feblen der Sehnenreflexe und des Plantar- und Abdominalreflexes. Verlangsamung der Schmerzleitung; exquisite Ataxie und Maskelsinnstörang. Schwanken bei Augenschluss; atactischer Gang.

Urinretention machte am ersten Tage die Catheterisation nöthig; der Urin leicht träbe, verhält sich mikroskopisch wie bei Cystitis.

Die inneren Organe bis auf die Leber normal; letztere ist etwas vergrössert (Sohnürleber), besitzt als Anhang, entsprechend der Lage dor 
Gallenblase einen deutlich palpabeln, nicht druckempfindlichen, birnförmigen Tumor.

16. Juni. Etiche Male Erbrechen unter kolikartigen, vom Hypogastrium rach der Magengegend heraufziehenden Schmerzen, Brennen im Epigastrium und starkem Durstgefühl.

Das Erbrochene giebt mit Methylviolett deutliche HCl-Reaction und enthält 0,19 pCt. freie Säure.

17. Juni. Das Erbrechen dauert fort. Säuregehalt 0,14 p C t. Temperatur normal; Pulsfrequenz 84. - Uricretention.

18. Juni. Urinretention, Obstipation. - Erbrechen, Magenschmerzen, Durst, Jactation, Schlaflosigkeit. Die Kranke bekommt Morgens $11 / 2$ Kaffeelöffel Karlsbader Salz und Abends 0,01 Morphium.

Vom 15.-21. Juni lässt das Erbrechen nach. Spontane Urinentleerung.

21. Juni Abends. Erbrechen, - Ord.: Antifebrin 0,25.

22. Juni. Temperatur $36,9^{\circ}$. Puls 72. - Erbrechen mit den frühoren Erscheinungen. Menge des Erbrochenen 200 Cbetm.; dasselbe ist stark saver und gallig gefärbt. Die Kranke bekommt Abends 0,5 Antifebrin.

23. Juni. Temperatur 37,1 ${ }^{\circ}$. Pulsfrequenz 80 ; häufiges Erbrechen in der Nacht mit den erwähnten Erscheinungen. Ord.: Antifebrin 0,75 Morgens und 0,5 Abends.

24. Juni. Kein Erbrechen in der Nacht; Temperatur Morgens $37,5^{\circ} \mathrm{C}$, Abends $38,1^{\circ}$.

Die Kranke nimmt vom 24.-30. Juni zwei Mal täglich Antifebrin 0,5; die Temperatur schwankt während dieser Zeit zwischen $37,0^{\circ}$ und $37,7^{\circ} \mathrm{C}$. Das subjectire Befinden und Ausseben der Kranken ist dabei ganz gut; sie kann aufstehen und umbergeben, isst mit vortrefflichem Appetit. Nur am 28. Juni litt sie an lancinirenden Sehmerzen in beiden N. ulnares. Kein Erbrechen mehr.

1. Juli Morgens. Temperatur 36,7; Pulsfrequenz 84. Wohlbefinden. Um 5 Uhr Nachmiltags Scbüttelfrost mit Darst. Abendtemperatur $39,3^{\circ}$ C., Prals 96. Der objective Befund unverändert.

2. Juli. Schlaf unruhig; Magendrücken, Durst. Bei gleichbleibender Behandlung bleibt die Temperatur vom 2.-4. Morgens zwischen 37,40 und $38,0^{\circ} \mathrm{C}$. Ausser den gastrischen Symptomen hatte sie vorübergehend lancinirende Schmerzen in den Beinen.

4. Juli. Temperatur $37,4^{\circ}$ C.; Puls 80 .

Abends Temperatur $40,0^{\circ}$; Puls 96 . Die Kranke sieht wieder ganz verfallon und am ganzen Körper blassgelb ans. Die Haut ist welk und trocken. Der Leib ist weich, nicht aufgetrieben; Gurren ist nicht zu erzeugen. Milzdämpfurg nioht vergrössert.

Die untere Lebergrenze steht unterhalb des Nabels und bat die vergrösserte Gallenblase als Anhang.

Die subjectiven Beschwerden sind Appetitlosigkeit, Uebelkeit, starker Durst und Magenschmerzen. 
Vom 5.-28. Juli fieberhafte Erkrankung. Die Fiebercurve entspricht der eines mittelschweren Typhus; in diesen Schranken wurde die Temperatur jedoch bei einer täglichen Verabreichung ron 1,0-1,75 Antifebrin gehalten. Vom 8.-11. Juli mehrere diarrhoische, intensiv gallig gefärbte Stühle; ganz vorübergehend war der Leib einmal aufgetrieben und eine leichte Vergrösserung der Milz zu constatiren. Alle äbrigen, auf einen Ileotyphus hinweisenden Symptome fehlten während der ganzen Dauer des Fiebers. Im Vordergrund standen zur Zeit des Fiebers wieder die gastrischen Krisen, die fast keinen Tag ausblieben, mit Vorliebe unter Magenschmerzen, Durst Brennen im Epigastrium etc. sich dann einstellten, "wenn's Fieber kam". Das Erbrochene war stets sauer, stark gallig gefärbt, auch zu der Zeit, wo sich ein intensiver Ioterus ausbildete, sowie bei dessen Abnahme. Der Icterus verlief unter starker Anschwellung und Druckempfindlichkeit der Leber, obne dass der Urin morphologisebe Bestandtheile wie bei Nephritis enthielt. Derselbe enthielt stets kleine Mongen Albumin, war trübe bei saurer Reaction.

Am 21. Juli erbrach sie zum letzten Male, pom 24. Juli ab fühite sie sich wohl; am 28. Juli wurde sie erst fieberfrei.

Vom 2.-7. August erneute gastrisehe $\mathrm{Kr}$ isen von ungemeiner Heftigkeit. Dabei blieb sie frei von Icterus; die Temperatur zeigte in den letzten Tagen des Spitalaufenthalts wieder Tendenz in die Höho zu gehen, wenn auch $37,7^{\circ} \mathrm{C}$. nicht überschritten wurde.

Von Antifebrin wurde dies Mal abgesehen, da es keine evidente, das Erbrechen sistirende Wirkung gezeigt hatte und statt seiner Cocain verabreicht; dasselbe hatte gar keinen Effect; Morphium allein half über die schlimmsten Anfälle hinweg.

7. August. Im Laufe des Vormittags nach vorausgegangenem Erbrechen mit Gastralgie drei Anfälle von Bewusstlosigkeit mit clonisob-tonischen Krämpen in den Armen und den Beinen; Verdrehen der Augen, Nackensteifigkeit. Pupillen weit, starr. Euuresis bei jedem Anfall.

Von dem Tage ab fühlte sich die Kranke ganz wohl; das Erbrechen war verschwunden.

10. August. Auf Wunsch entlassen.

\section{Fall IV.}

Vor 10 Jahren Syphilia, Frkältungen, angestrengte Thätigkeit. Seit $31 / 4$ Johren Digestionsbeschwerden, lancinirende Sohmerzen, Paraesthesien. Seit 2 Jahren Abnahme des Körpergewiohts und der Träfte, Urinbeschwerden; Kopfohmerz, Larynxkrisen leichter Art. Abnahme des Geschlechtstriebes. - Leichte psychische Veränderung, reflectorische Pupillenstarre. Schwanken oc. cl., Sehnenreflexe fehlend oder abgesohwächt. Cystitis. Bedeutende Besserung durch Schmiercur und faraw 
dische Pinselung. - Suproorbitalneuralgie, Kopfschmerz, Schlaflosigkeit. Digestionsbeschwerden, gastrische Krisen, Salivation, Finterorrhoen, Larynxkrisen, Irregularitat des PuIses, Fieber centralen Ursprungs.

Rudolf Weinmayer, 32 Jahre alter Locomotivfübrer von Kaiserslantern, rec. 20. September 1885 .

Keine neuropathische oder sonstige Belastung. Als ursächliche Momente der jetzigen Krankheit sind zu erwähnen: häufge Erkältangen, angestrengte Thätigkeit und Syphilis, die er sich im 22. Jabre zuzog.

Vor 6 Wochen consultirte or Herrn Prof. Erb wegen Digestionsbeschwerden. Folgende anamnestische and objective Erscheinungen liessen dieselben nur als eins dor Symptome der ejgentlichen Krankheit erkennen.

Seit $3 \frac{1}{2}-4$ Jabren Appetitlosigkeit; Unruhe, Kollern, Druok und Schmerz im Loibe; Iancinirende Schmerzen in beiden Beinen, Prickeln in den Füssen and Händen; seit einem Jahre starkes Müdigkeitsgefühl. Abnalumedes Geschlochtstriebes, trägo Urinentleerang. Abnahme des Körpergewichts um 30 Pfund innerhalb zwei Jahren. Roissen im Kopf, Kopfweb. Hustenanfälle alle $2-10$ Tage, eingeleitet darch Prickeln im Kehlkopf.

An dem Kranken fiel zanächst sein eigenthümliohes Benehmen auf; ging er zerstreut im Zimmer auf und ab and gab Antworten, die nicht zur Sache gehörten.

Pupillen verengert, ungleich weit, fast starr. Augenmuskeln und Augenhintergrund normal. Schwanken bei Augenschluss, unsicheres Stehen auf einem Fusse. Kehrtmachen schlecht. Gang leicht stamp fend, mit dem rechten Beine schleudernd.

Grobe motorische Kraft durchweg gut; ausser der allgemeinen Abmagerung bestehen keine trophischen Störungen.

Der Tastsinn und die Tastsinnleitung vollständig gut am ganzen Körper.

Die Schmerzempfindung ist am Thorax rom Schultergürtel abwärts horabgesetzt, mehr noch an den oberen Extremitäten ond fehlt fast ganz am rechten Bein, während das linke noch etwas besser empfindet.

Der Temperatursinn ist nur an den Unterschenkeln and Oberschentreln herabgesetzt.

Die Sehnenreflexe sind am rechten Arm und rechten Bein erlosehen, dagegen links erhalten.

Plantar- und Bauchreflex beiderseits lebhaft; der Cremasterreflex fehlt. Muskelsinn der Beine vermindert; Ataxie des rechten Beines.

Die inneren Organe normal.

Urin trüb, neutral, enthält Spuren von Eiweiss; Eiterkörperchen.

In der letzlen Zeit konnte der Kranke kaum mehr 10 Minuten Iang gelhen, ohne dass er wegen Uebermüdung sich setzen musste.

Am 22. Februar 1886 verliess der Kranke die Klinik wieder. Er hatte während dieser Zeit 77 Einreibungen mit Ung. cin, 3,0 gemacht, war täglich nach der Methode von Ramp f elektrisirt worden. 
Eine am 6. Decemler vorgenommene faradocutane Sensibilitätsprüfung bestätigte den oben notirten Befund. Bei ganz normalem Tastgefühl bestand stets Verminderung des Schmerzsinns, nur Spitzo und Kopf der Nadol komnten. an den Beinen nicht scharf aliseinander gehalten werden.

Während des Auferithalts in der Klinik klagte er über Spannen der Kopfhaut, Reissen im $\mathrm{N}$. supraorbital. sin., eingenommenen Kopf. Magendruck, Kollern und Druck im Leibe. Stuhldrang, Uebolkeit, Appetitlosigkeit, vom 22.--24. December tägliches Erbrechen. Das Erbrochene reagirte sauer uud gab die Salzsäurereaction; vorübergehend Diarrhoen mit Stubldrang. -- Urinentleerung träge; stots schwacher Blasencatarrh. - Hustenreiz mit Bitzel im Halse und ca. 10 Minuten anbaltenden Hustenparoxysmen.

Es war während der Belandlung eine sehr beträchtliche Besserung eingetreten. Die Pupillen waren weiter geworden, reagirten auf Licht ziemlich prompt; fast kein Schwanken mehr bei Augenschluss; die Ataxie war ganz geschwundeu. Die Sensibilität eto. nicht merklich verändert.

Der Kranke hatte 3,5 Kilo an Gewicht $z$ ugenommen, ging stundenlang im Garten spazieren und fühlte sioh so kräftig, dass or glaubt, 4-5 Stunden ohne Unterbrechung gehen zu können.

Bei seiner Entlassung bekam er Kal. jodat. mit.

Am 13. November 1886 liess sich der Kranke wieder in die Klinik anfnehmen wegen eines acuten Blasencatarrhs, den or sich im October zugozogen hatte durch eine Erkältung. -

Er giebt an, dass er schon 3 Wochen nach seiner Entlassung im Früh jahr seinen Dienst wieder antrat und denselben mit nur zweimaliger Unterbrechung von je 14 Tagen bis jetzt vollständig versah. Er konnte 4-5 Stunden am Tage gehen, ermüdete datei etwas rascher als früher. Die früheren Beschworden bestanden in geringer Intensität weiter.

Die objective Untersuchung ergab, dass die Pupillen etwas träger reagirten als früher, dass rom 4.-6. Intercostalraum Hyperästhesie gegen Kälte bestand, und dass bei starker Herabsetzung des Temperatursinns an dea Beinen eine falsche Perception insofern vorhanden war, als warme Gegenstände stets den Eindruckvonkalten hervorriefen. Die Sohmerzleitung war bis zu 5 " verlangsamt.

Die Behandlung richtete sich gegen den Blasencatarrh (Borwasserausspülungen).

Bis zum 10. December war der Kranke fieberfrei, litt an Appetitlosigkeit, Uebelkeit, Magendruck, Enteralgien mit Diarrhoen; die letzten daverten unter starkem Drang 1-3 Tage.

Vom 11. - 23. December fieberte der Kranke ziemlich hoch, so. dass Antipyrin per Klysma gegeben wurde, weil er meist Brechneigung verspürte, läufig erbrach und stets Kollern und Druck im Leibe empfand.

16. December Morgens. Temperatur 38,6, Puls 120. Patient erbrach in der Nacht, hatte 6 diarrhoische Stüble unter starken Drang. 
Verfallenes Aussehen; Abgeschlagenheit; heute Nacht 3 Uhr merkte Patient, dess er die rechte Hand nicht dorsalwärts bewegen kronnte. Die Untersuchung ergiebt Parese imGebiet des recbten $\mathrm{N}$. radialis, die wohl in Folge eines Druckes entstanden war. Während des Erbrechens und der Zeit des Brechreizes hing der Kranke, ganz wie Kranke mit gastrischen Krisen, mit der Hand an dem seitlich rom Belt stehenden Stuhl und legte den Kopf auf den Oberarm.

20. December. Heiserkeit und Gefühl im Halse, als ob etwas in der Luftröhre stecke. Während der ganzen Zeit des Fiebers litt er an Erbrechen, Salivation, Diarrhoen. Eine Ursache für das Fieber konnte nicht aufgefunden werden. Der Leib war eingesunken, bis auf die Wirbelsäule und seitlich leicht abzutasten; lreine Milzvergrösserung. Urinentleerung und Urin wie zur Zeit, wo er nicht fieberte.

Da nach einem fieberfreien Intervall von 5 Tagen die Temperator Abends $1 / 2$ Stunde nach einer Blasenausspülung $38,8^{\circ} \mathrm{C}$. betrug, wurde ron jetzt ab von Blasenausspülungen abgesehen. 3 Tage später war er wieder fieberfrei. Am nächsten Tage 1. Januar $1887 \mathrm{stieg}$ die Temperatur wieder olne belannte Ursache und es schloss sich nun ein hartnäekiges Fieber an mit unregelmässigem Verlauf, das bis zum Austritt des Kranken aus dem Spital am 6. März 1887 andauerte, und nur selten bei fortgesetztem innerlich Gebrauch von Antifebrin 1,25-1,5 pro die in drei Dosen zur Norm herabgedrückt wurde. Weder an den intrathoracischen, noch an den intraabdominalen Organen liess sich während dieser Zeit eine Erkrankung nachweisen. Für Typhus abdominalis sprach nichts; eine Gelenkaffection, ein Abscess etc. war nicht aufzufinden. Im Vordergrund standen Magen- und Darmerscbeinungen. Die Symptome, die sich, so weit sio vom Magen herrühren, so gut wie täglich zeigten, so weit sie vom Darm, dem Kehlkopf und dem Herzen ausgingen, resp. da ausgelöst wurden, seltener kamen, sind kurz zusammengefasst: Appetitlosigkeit, Uebelkeit, Brechreiz, Erbrechen, pappiger Geschmack, Druck in der Magengegend. Das Erbrochene enthält Salzsäure; eine qrantitative Bestimmung ergab 0,08 pCt. Säure. Enteralgien, Druck im Hypogastrium, zeitweise Diarrhoen, dann Stuhlgang bei festem Stuhl; Salivation; in 24 Stunden worden bis zu $300 \mathrm{Cbctm}$. Speichel abgesondert. Kitzel im Kehlkopf, „als ob etwas darin stecke". Der Puls setzte zuweilen aus oder war jrregulär; auffallende Röthe der Haut.

Urinbeschwerden, Cystitis.

Kopfweh und manchmal Schwindel, ferner Schlaflosigkeit, wogegen anch zur Zeit, in welcher der Kranke nicht fieberte, Urethan mit vorübergehendem Erfolg verabreicht worden war.

Am 3. Februar wurde nochmals eine Schmiercur eingeleitet, die den Krankheitsverlauf nicht wesentlich änderte, aber eher einen günstigen Einfluss auf das Allgemeinbefinden und das Fieber zu haben schien. Der Kranke stand in der letzten Zeit auf und lronnte ganz gut gehen. 


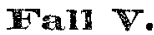

Frkältungen. 1870 Geschwür am Penis. Seit zwei Jahren lancinirende Schmerzen; seit $3 / 4$ Jahren Schwindelanfälle, Gedächtnissschwäche, Taubheițgefühl in den Armen und Beinen, Unsicherheit im Gehen, Abmagerung: der Hände. - Reflectorische una sccommodative Pupillenstarre. Tastempfindung herabgesetzt. Beiderseits Atrophie der kleinen Handmuskeln mit FaR. - Patellar- und Achillessehnenrefieze fehien; Hypalgesie der Beine.

Franz Heeg, 49 Jahre alter, verheiratheter Colporteur von Neustadt, weiss über seine Eltern und Geschwister siohere Angaben nicht zu machen. Seine Frau gebar drei gesunde, noch lebende Kinder und blieb selbst stets gesund.

Den Feldzug von $1870 / 71$ machto er als Marketender mit, erkältote sich oft, zog sich damals anch eine Gonorrhoe und ein Geschw ür am Glied zu, das der Beschreibung nach mit Cupr. sulf. behandelt wurde und keine syphilitischen Erscheinungen direct zur Folge hatte.

Seit 2 Jahren leidet er an lancinirenden Schmerzen; seit $3 / 4$ Jahren wird er von karzdauernden $\mathrm{Sch}$ windelanfäll en heimgesucht, die Kopfschmerz hinterlassen; damit rergesellschaftet sich Gedächtnissschwäch $\theta$. Ausserdem nahm er noch an sich wahr Taubheit aller 4 Extremitäten und Atrophie der kleinen Handmuskeln beiderseits, ferner Unsicherheit im Gebon. Keine lrankhaften Erscheinungen seitens der Sphincteren.

Bei der klinischen Vorstellung warde constatirt: Reflectorische und accommodative Pupillenstarre bei ziemlich weiten Pupillen. Intelligenz gut. Unsichere Tastempfindung der oberen Extremitäten. Atrophie des Thenar und Hypothenar der linken und mehr noch der rechten Hand; ausserdem sind einzelne Musc. interossei stark abgemagert; die abgemagerten Muslreln geben bei ziemlich starken Strömen tbeils partielle, theils complete EaR. Die Sehnenreflexe der Arme sind erhalten, keine Ataxie.

An dem Rumpf und den unteren Extremitäten ist bei gut erhaltener Tastempfindung der Schmerzsinn herabgesetzt; daneben besteht $\mathrm{Hy}$ perästhesiegegen Kälte und verlangsamte Schmerzleitung.

Patellar- and Achillessehnenreflexeerloschen. Hautreflexe normal. Leichto Coordinationsstörung mit Unsicherheit des Muskelsinns.

Der A ugenhintergrund wird normal befunden. Dio Hörfähigkeit ist beiderseits herabgesetzt.

Die inneren Organe normal. Urin eiweissfrei, sauer, klar, specifiscbes Gewicht 1019.

Der Kranke blieb nur so kurze Zeit in der Klinik, dass ein Erfolg von der eingeleiteten Behandlung nicht zu sehen, aber auch nicht zu erwarten war. 
In den soeben mitgetheilten Fällen beherrschen, mit Ausnahme des fünften, Erscheinungen seitens des Digestionstractus das Krankheitsbild und gaben den Kranken die erste Veranlassung, ärztlichen Rath einzaholen.

Die Kranken II. und III. hatten unter schweren, 2-4 Wochen ohne Unterbrechung tagtäglich wiederkehrenden gastrischen Krisen mit den gewöhnlichen Symptomen schwer zu leiden. In allen Positionen konnte man sie in ihrem Bett antreffen, ein Bild des Jammers und Elends. Neben den heftigsten, vom Hypogastrium nach der Magengegend sich herauf wälzenden Schmerzen wurden am schwersten empfunden das starke Brennen im Magen, der unstillbare Durst, die unaufhörliche Brechneigung mit dem bäufigen Erbrechen, die Eingenommenheit des Kopfes und die allgemeine Abgeschlagenheit. Die letztgenannten Symptome finden sich nach den neueren Erfahrungen bei Magenkrankheiten mit Hyperacidität und Hypersecretion des Magensaftes. Die Branken I. und IV, litten weniger durch gastrische als durch enterische Krisen, die in längeren Intervallen für kürzere Zeit sich bei hnen einstellten.

Durch die Sahli'sche Publication *) über das Vorkommen abnormer Mengen freier Salzsäure im Erbrochenen eines mit gastrischen Krisen behafteten Tabischen wurde ich veranlasst, das Erbrochene genauer zu untersuchen. Wie die Tabellen in der Krankengeschichte II. zeigen, bestand während der ganzen Zeit der Krisen Hypersecretion des Magensaftes mit zejtweiser Hyperacidität. Der Salzsäuregehalt wechselte, erreichte häufig das Doppelte des Normalen, sank nur selten bei ganz copiösem Erbrechen unter die Norm. Ein An- und Absteigen des Salzsäuregehaltes, parallel der Heftigkeit der Krisen, konnte ich nicht constatiren. Allerdings kommt dabei in Betracht, dass mit den heftigsten Brechanfällen anch das Brennen im Magen und der Durst zunimmt, dessen Besänftignng durch reichlichere Zufuhr von Flüssigkeit nothwendigerweise eine Verdünnung des Magensaftes und der denselben zusammensetzenden Bestandtheile zur Folge hat. Der Magensaft dieses Kranken enthielt nur Spuren von Milchsäure, war meist frei davon. - Im Fall III. wurde das Erbrochene nur 2 Mal untersucht; es enthielt 0,19 und 0,14 pCt. Säure, gab aber ausser der Salzsäurereaction auch diejenige der Milchsäure. Bei der Kranken IV. enthielt das Erbrochene nur 0,1 pCt. freie Säure.

") Correspondenzblat für schweizer Aerzte 1885. No. 5 . 
So viel ich in der Literatur sehe, ist bis jetzt nur vereinzelt dem Verhalten des HCl-Gehaltes des Erbrochenen Tabischer Aufmerksamkeit geschenkt worden. Sahli war wohl der erste, der auf einen hohen Salzsäuregehalt des Erbrochenen aus der starken Säurereaction schloss. Quantitative Untersuchungen hat er nicht angestellt. Der Säuregebalt soll bei späteren Krísen nicht mehr abnorm gewesen, also gesunken sein. Rosenthal*) fand in zwei Fällen von Tabes vom 3.-5. Tage der Brechperiode, die 6-12 Tage dauerte, Salzsänremengen von $0,30-0,32$ pCt., ferner Spuren von Milchsäure; in den letzten Tagen nur $0,095-0,1$ pCt. Sodann hat Simonin**) nach der Angabe Lannois"***;) Hyperacidität des Erbrochenen nur passager mit den Krisen auftreten sehen; das Maximum des Säuregehaltes soll mit den heftigsten Krisen zusammengefallen seìn. Ferner glaube ich gelesen zu haben, dass Ewald Salzsäure in dem Erbrochenen eines Tabischen vermisste.

Ausser durch die Hyperacidität und Hypersecretion des Magensaftes ist der Fall II. noch bemerkenswerth durch die ausserordentlich lange Dauer der Brechperioden, von denen eine über 4 Wochen dauerte. Nach Fournier $\downarrow$ ) sollen dieselben nur ausnahmsweise eine Wocheüberschreiten, während Sabli und Rosenthal von 6 bis 12 Tagen sprechen.

Aber nicht allein die Secretion des Magensaftes war paroxysmatisch gesteigert, sondern auch die Speichelabsonderung (Fall I., II., IV.) und die Secretion seitens der Darmschleimhaut (Fall I. und IV.). Wenigstens glaube ich die periodisch auftretenden Enterorrhoen, die auch längst durch andere Autoren beschrieben sind, in dieselbe Categorie rechnen zu müssen.

Wie ist im Fall III. der Icterus aufzufassen? Handelt es sich um eine Störung, die die gleiche Ursache wie die Secretionsanomalien der genannten drüsigen Organe hat oder um eine zufällige intercurrente Leberaffection oder eine sonstige Complication. Um einen gewöhnlichen Staungsicterus hat es sich wobl nicht gehandelt; dagegen sprechen ausser den gallig gefärbten Stühlen, auch die erbrochenen gallig gefärbten Flüssigkeitsmengen zur Zeit, wo die Kranke intensiv icterisch gefärbt war. Viel mehr Aehnlichkeit hatte die mit hohem continuir-

*) Ueber nervöse Gastroxie. Wiener med. Presse 1886. No. 15-17. **s) Limonin, Thèse de Lyon 1886.

***) Lannois, Sécrétion gastrique. Revue de médéc. 1887. No. 5. pag. 433.

†) A. Fournier, De l'ataxie locomotrice. 1882. pag. 136. 
lichen Fieber verlaufende Affection mit jener acuten Infectionskrankheit, die A. Weil*) vor nicht langer Zeit beschrieben hat. Weil neigt dazu, seine Fälle als Abdominaltyphus mit Icterus und Nephritis aufzufassen, lässt aber anch der anderen Auffassung ihre Berechtigung, dass es sich um einen „Morbus sui generis" handelt. Von den Weil'schen Fällen, die ich zum Theil aus eigener Beobachtung kenne, differirte das Krankheitsbild nur durch das Fehlen der Nephritis. Ich glaube deshalb, dass es sich hier um einen Icterus bei einer intercurrenten Infectionskrankbeit und nicht um ein Symptom der Tabes handelt.

Im Hinblick auf das Fieber erscheint der differential-diagnostische Excurs vielleicht überflüssig. Weniger überflüssig wird die Erörterung erscheinen, wenn man den Fall IV. mit in die Betrachtung hereinzieht. Hier haben wir ein Fieber, das in keiner Weise Aehnlichkeit hat mit dem irgend einer Infectionskrankheit. Nach 7 tägigem Fieber (bis zu $40^{\circ} \mathrm{C}$.) sehen wir eine ebenso lang andauernde fieberfreie Periode, dann einige Tage leichtes descendirendes Fieber, wieder einen fieberfreien Tag, dem dann eine 9 wöchentliche Fieberzeit folgt, während der nur 3-4 Mal ein bis drei durch Antifebrin herbeigeführte, fieberfreie Tage liegen. Dabei befand sich der Kranke subjectiv ziemlich wohil. Da die genaue Untersuchung der inneren Organe, wobei auch die Exploration per rectum nicht versäumt wurde, irgend welche pathologische Veränderung, die man für das Fieber verantwortlich machen konnte, nicht erkennen liess, blieb nichts übrig, als die Ursache des Fiebers anderswo zu suchen oder sich damit zu begnügen, eine febris e causa ignota anzunehmen. Ich wählte den ersten Weg und kam zu dem Schluss, dass wir es hier aller Wahrscheinlichkeit nach mit einem Fieber centralen Ursprungs zu thun haben. Weniger der stets negative Befund der inneren Organe, der an und für sich noch nichts beweisen würde, als vielmehr die Begleiterscheinungen des Fiebers, die Magen- und Darmerscheinungen, die Salivation, die starke Beschleunigung (bis 140), die Arrhytmie und das Aussetzen des Pulses, der Kitzel im Larynx mit Hustenreiz, das wenn auch seltene Beklemmungsgefübl anf der Brust führten mich zu der obigen Annahme.

Ich brauche nur an die agonalen und postmortalen Temperatursteigerungen bei Gehirnkrankheiten zu erinnern oder die rasche Tem-

*) A. Weil, Ueber eine eigenthümliche, mit Milztumor und Nephritis einhergehende acute Infectionskrankeit. Deutsches Archiv für klinische Medicin. Bd. 39. 
peratursteigerung nach Verletzung des Halsmarks und die in gleichem Sinne ausgefallenen Experimente an Thieren anzuführen, um eine Stütze für obige Auffassung zu gewinnen.

Nimmt dieser Fall das Interesse durch die Temperatursteigerung für sich in Anspruch, so sind Fall II. und III. durch die epileptiscben Anfälle noch bemerkenswerth, die im Fall II. zwei Mal, im Fall III. 3 Mal in kurzen Intervallen einander folgten. Sie sind durch Bewusstlosigkeit, Reactionslosigkeit der Pupillen, clonische und clonischtetanische Zuckungen etc. als solche genügend gekennzeichnet. Interessant bezüglich der Genese derselben sind die Prodromal und Begleiterscheinungen im Falle II. Die hauptsächlichsten derselben waren die einen vollen Tag vorausgehende Irregularität des meist beschleunigten Pulses, die unregelmässige Athmung, der rasche Wechsel der Gesichtsfarbe mit passagerer Röthung des Thorax. Unter starker Blässe des Gesichts und noch beträchtlicheren Schwankungen des Pulses und der Respiration stellen sich dann die epileptischen Krämpfe mit Bewusstlosigkeit ein, die unter starker Röthung des Gesichts wieder schwinden. Der Zustand des Kranken flösste mir Besorgniss ein und wie mich später der 4. Fall von Leyden*), der unter ähnlichen Symptomen zu Grunde ging, lehrte, mit Recht.

Fragen wir nach dem Ausgangspunkt aller dieser Erscheinungen, so kommt zunächst das verlängerte Mark in Betracht. Hier liegen die Centren für die normale geregelte Thätigkeit aller der Organe zusammen, die wir in den obigen, wie in vielen sonstigen von anderer Seite beschriebenen Fällen gestört fanden. Hier liegen die Vaguscentren, die Centralorgane des secretorischen und vasomotorischen Systems u. s. w. Ob man neben diesen noch eigene calorische Centren annebmen oder die Aufgabe der Wärmeregulirung dem vasomotorischen übertragen will, wofür nach v. Recklinghausen ${ }^{* *}$ ) die Gründe überwiegen, kommt hier wenig in Betracht. Das vasomotorische Centrum in der Medulla oblongata spielt aber.nach Kus maul auch die Hauptrolle beim Zustandekommen der Epilepsie, die wir hier bei Tabischen, die früher nicht an Epilepsie gelitten hatten, zu gastrischen Krisen hinzutreten sehen. Solche Fälle beweisen auch die Richtigkeit der Nothnagel'schen Meinung**; )dass verschie-

*) Leyden, Ueber Herzaffectionen bei der Tabes dorsalis. - Centralbl. für klin. Medicin. 1887 No. 1 .

***) v. Recklinghausen, Allgem. Pathologie des Kreislaufs S. 460 u.ff. ***) Nothnagel, Epilepsio und Eclampsio in v. Ziemssen's Handbuch der Pathologio und Therapie. Bd. XII. 2. 
denartige anatomische Alterationen den sie (die Epilepsie) bildenden Symptomencomplex hervorrufen können, vorausgesetzt, dass diese Alterationen immer die gleichen (anatomisch oder auch physiologisch gleichwerthigen) Partien in Brücke und verlängertem Mark treffén“.

Ausser den erwähnten Symptomen sprechen auch die Schwankungen des Pulses und der Respiration, die Salivation etc., dafür, dass für gewöhnlich die geschilderten Symptome eine centrale Ursache haben. Da man es in der Mehrzahl der Fälle mit Reizvorgängen zu thun hat, die die Centren nur paroxysmatisch zu krankhafter Thätigkeit antreiben, während sie, soweit ersichtlich, in der Zwischenzeit wieder normal arbeiten, darf man auch nicht erwarten, dass regelmässig grob anatomische Veränderungen derselben bei der Autopsie gefunden werden. Ich brauche nur an die functionellen Neurosen, die Epilepsie, die Neurasthenie, Hysterie (mit ihrem monatelangen Erbrechen) etc. zu erinnern, um zu zeigen, wie weit wir in der Erkenntniss der feineren Vorgänge im Nervensystem, die so prägnante Symptome setzen, noch zurück sind. - Für stabile Erscheinungen, wie Lähmungen mit Atrophien etc. darf man ein anatomisches Substrat mit mehr Sicherheit erwarten.

Zweifellos kann die Erkrankung peripherer Nerven ähnljche, wenn auch nicht in jeder Beziehung gleiche Erscheinungen bewirken, wie diejenige ihrer Centren. So kann z. B. der Vagus in einem solchen Krankheitsfalle in seiner specifischen Function mit Vagus- und Herzkrisen etc. antworten, wie ein gewöhnlicher sensibler Nerv mit Schmerzparoxysmen. Aber wie eine gewöhnliche Neuralgie ohne objective Sensibilitätsstörungen nỏch keineswegs eine anatomische Veränderung des Nerven, eine Neuritis, voraussetzt, ebenso wenig eine der genannten Krisen eine sichtbare Affection des Vagus und seiner Centren.

Finden sich auch bei der Obduction Veränderungen der Nerven, so ist durchaus noch nicht ausgemacht, dass dieselben auch für die intra vitam beobachteten Symptome ohne Weiteres verantwortlich gemacht werden dürfen. Sahen doch Oppenheim und Siemerling*) Nervendegeneration bei solchen Individuen, die während des Lebens gar keine motorischen und sensiblen Störungen boten. Die Veränderungen waren „gering“, „mittleren Grades“, „nicht unbeträchtlich“, doch waren sie „nie so mächtig, wie bei der Tabes oder von Fällen schwerer multipler Neuritis. Die beiden Autoren sagen selbst, dass eine völlige Congruenz zwischen klinischen Ausfallserscheinungen

*) Oppenheim und Siemexling, Beiträge zur Pathologie der Tabes dorsalis otc. Dieses Archiv Bd. XVIII. 1. und 2. Heft. 
und anatomischer Läsion nicht zu bestehen scheine, und dass für die Schwere der klinischen Symptome vor Allem auch die Acuität, mit der sich die degenerativen Processe entwickeln, von Bedeutung sei. Auch die Bedeutung der Acuität darf jedoch nicht überschätzt werden, wenn man bedenkt, dass die beiden Typhuskranken mit Nervendegeneration mässigen und mittleren Grades in der zweiten resp. dritten Woche starben, somit die Nervendegeneration nicht mehr Zeit hatte zur Entwickelung wie eine rheumatische Neuritis.

Oppenheim und Siemerling sind mit Recht in ihren Schlüssen sehr vorsichtig und die Vorsicht ist auch bei Abwägung der anatomischen und klinischen Befunde sehr geboten, denn nach dem jetzigen Stande der Dinge ist es mehr oder weniger dem Belieben des einzelnen Untersuchers anheimgestellt, anzunehmen, ob Nervenveränderungen leichteren und mittleren Grades die Ursache des Krankheitsbildes waren oder nicht.

Was nun speciell den Befund bei gastrischen etc. Krisen betrifft, so haben Kahler und Andere eine Erkrankung der Vagus-Accessoriuskerne, 0 ppenheim und $\mathrm{Krauss}$ Atrophie des N. vagus und des N. recurrens als zweifellos constatiren können.

Der Kranke IV. litt ferner an einer leichten Lähmung des rechten N. radialis. Dieselbe ist in diesem Falle eine periphere und zwar eine Drucklähmung und als eine zufällige zu betrachten, wenn man nicht nach dem Vorgangevon Oppenheim und Siemerling (l. c.) annehmen will, dass Tabische und Alkoholisten wegen sich latent bei ihnen abspielender Nervenveränderungen besonders zu solchen Lähmungen disponiren. Für eine spontan entstandene periphere Lähmung halte ich sie wegen der anamestischen Erhebungen nicht. Ist dies also eine periphere Lähmung, so beruht im Fall V. die mit EaR verbundene Parese und Atrophie der kleinen Handmuskeln, die beiderseits ohne Auswahl eines Nervengebiets ergriffen sind, nach unserer heutigen klinischen, durch vereinzelte anatomische Befunde gestützten Anschaung auf centraler Ursache. Charcot, Leyden und Westphal constatirten in Tabesfällen, die mit amyotrophischer Lähmung einhergingen, Degeneration in den grauen Vorderhörnern des Rückenmarks und in den Kernen der Medulla oblongata. Klinische Beobachtungen, die hierher gehören, theilt A. Fournier in seinem citirten Werk über syphilitische Tabes mit, ferner hat Eulenburg*)

*) Eulenburg, Ueber Combination von Tabes dorsalis mit motorischer Systemerkrankung des Rückenmarks. - Deutsche medic. Wochenschr. 1887. No. 35. 
schon früher und wieder neuerdings solche Fälle publicirt. Unser Fall gleicht dem 2. Falle Eulenburg's, während dessen erster Fall schon weiter vorgeschritten ist. Der 3. Fall desselben Autors, ausgezeichnet durch vorwiegend rechtzeitige Muskelatrophie, Ophthalmoplegia externa etc., gehört in dieselbe Categorie. Desshalb, weil der Kranke Bleiarbeiter war, einen rückschreitenden Gang des Krankheitsprocesses anzunehmen, dass es sich also um „die Combination einer (auf saturniner Basis beruhenden) degenerativen Systemerkrankung des corticomusculären Leitungssystems mit degenerativer Hinterstrangsaffection" handele, halte ich nicht für richtig. Mag auch die Beschäftigung mit Blei eine Disposition zur Miterkrankung der Vorderhörner abgegeben haben; die ersten Krankheitssymptome waren die der Tabes dorsalis und zu ihnen gesellte sich erst die amyotrophische Lähmung. Ein Vergleich des Krankheitsbildes dieses Falles mit demjenigen der Fälle Charcot's*) und Westphal's**) spricht ebenfalls in diesem Sinne. Aller Wahrscheinlichkeit nach handelt es sich doch, wie auch $\mathrm{Charcot}$ richtig hervorhebt, um das Fortkriechen des Krankheitsprocesses von den Hinterhörnern auf die Vorderhörner des Rückenmarks und weder um eine Poliomyelitis saturnina, noch um eine Erkrankung des corticomusculären Leitungssystems, welch' beiden letzten Krankheiten klinisch wie anatomisch auseinander zu halten sind.

Therapeutisch ist in Fällen, in denen Syphilis unzweifelhaft vorausgegangen ist, ebenso gut wie bei anderen Krankheiten mit syphilitischer Vorgeschichte eine antisyphilitische Behandlung zu versuchen und dies um so mehr, wenn bis dahin eine solche noch gar nicht oder nur bei der ersten Infection in wenig energischer Weise vorgenommen wurde. Sind die Kranken sehr heruntergekommen, so wartet man mit einer Innunctionscur, bis sie bei einer anderen Behandlung, verbunden mit guter Ernährung, kräftiger geworden sind. Diese Vorsicht ist geboten, weil der Erfolg der Innunc. tionscur nicht vorauszusehen und Schwächung des Individuums so vie wie möglich $\mathrm{zu}$ vermeiden ist. $\mathrm{Zu}$ vorsichtig braucht man jedoch auch nicht zu sein, wie die Kranken II. und IV. beweisen, die trotz lange fortgesetzter Schmiercur an Gewicht während derselben zunahmen, was wiederum mit der Erfahrung übereinstimmt, dass durch Syphilis bedingte Abmagerung durch eine Schmiercur oder durch

*) Charcot, Leçons sur les maladies du system. nerveux. 1885. II. pag. 18.

**) Westphal, Dieses Archir Bd. XVIII. 3. 
464 Dr. J. Hoffmann, Beitrag zur Aetiologie, Symptomatologie etc.

Jodkaligebrauch schwinden kann. Jedenfalls widerlegen diese Fälle die Behauptung, dass die Quecksilbercur Tabischen stets schade. Jodkali ist selbstverständlich ebenfalls zu versuchen. Im Uebrigen bleibt die sonstige Behandlung der Tabes (Tonica, Nervina, Kaltwasserbehandlung, Elektricität etc.) zu Recht bestehen. Bei dem chronischen Verlauf der Tabes wird man dieselben mehr oder weniger in Anwendung bringen. Gegen die Schmerzen leistete Antifebrin und Antipyrin gute Dienste; dieselben wirken in verschiedenen Fällen in verschieden langer Dauer; in verzweifelten Fällen kommt man ohne Morphium nicht aus; doch gehören diese zu den Ausnahmen.

Bei den schweren gastrischen Krisen linderte Antifebrin, in Dosen von $0,5-0,75$ zwei bis drei Mal täglich verabreicht, die Schmerzen und führte die Kranken in einen angenehmen Schlummerzustand. Eine Sistirung des Erbrechens wurde ebensowenig damit erzielt, wie mit den anderen dagegen angewendeten Mitteln. 